Supporting Information

\title{
Low- to Room-Temperature Dehydrogenation of Dimethylamine Borane Facilitated by Ionic Liquids: Molecular Modeling and Experimental Studies
}

\author{
Debashis Kundu, Gopal Pugazhenthi and Tamal Banerjee* \\ Department of Chemical Engineering, Indian Institute of Technology Guwahati, Guwahati - \\ 781039, Assam, India \\ * Corresponding author: tamalb@iitg.ac.in
}
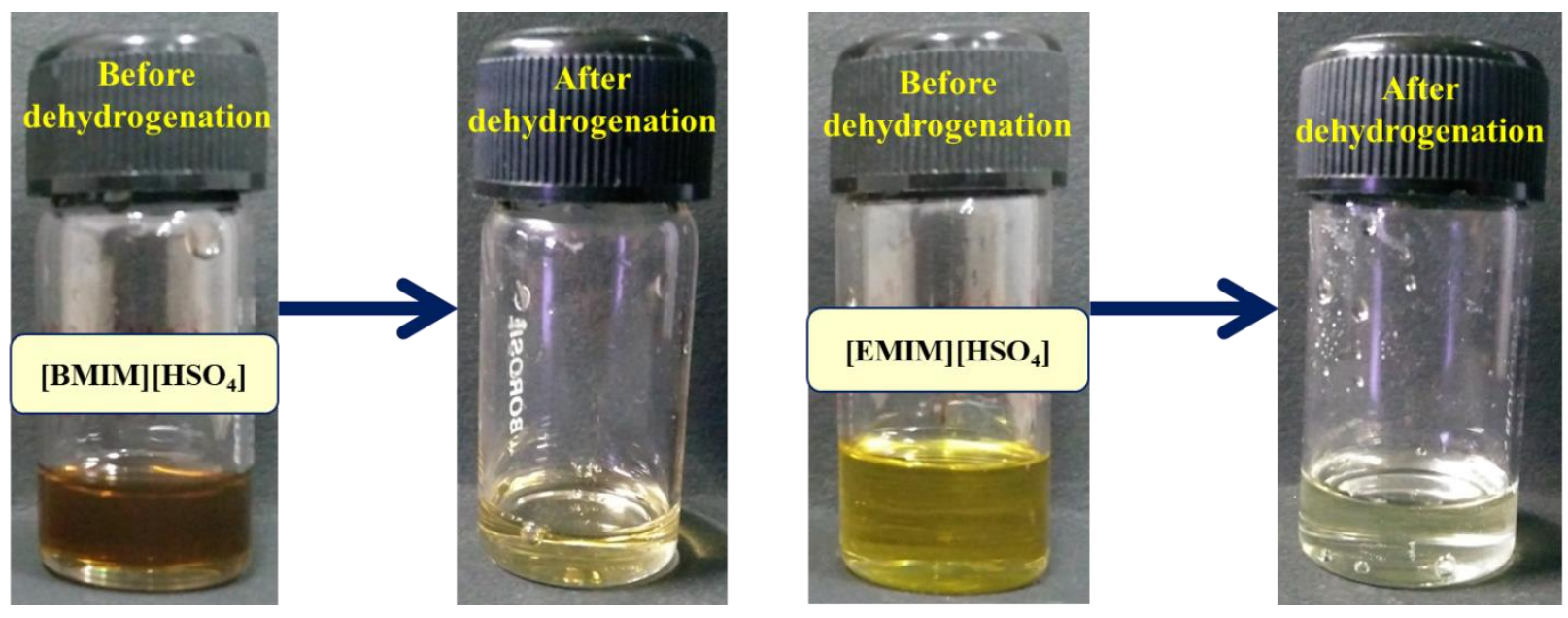

Figure S1. Digital image of ILs at before and after dehydrogenation 


\begin{tabular}{|l|l|}
\hline \multicolumn{2}{|c|}{ Table S1. COSMO-SAC predicted In(IDAC) } \\
\hline \multicolumn{2}{|c|}{ Ionic Liquid } \\
\hline
\end{tabular}




\begin{tabular}{|l|l|}
\hline 1-butyl-3-methylimidazolium tetrafluroborate & -0.85 \\
\hline 1-butyl-3-methylimidazolium thiocyanate & -1.05 \\
\hline 1-butyl-3-methylimidazolium tosylate & -1.90 \\
\hline 1-butyl-3-methylimidazolium trifluoromethanesulfonate & -0.80 \\
\hline 1,3-cyanopropyl-3-methylimidazolium bis trifluoromethylsulfonyl imide & -0.51 \\
\hline 1,3-cyanopropyl-3-methylimidazolium chlorine & -4.45 \\
\hline 1,3-cyanopropyl-3-methylimidazolium dicyanamide & -0.78 \\
\hline 1-decyl-3-methylimidazolium chlorine & -4.45 \\
\hline 1-decyl-3-methylimidazolium tetrafluroborate & -1.04 \\
\hline 1,3-diethoxyimidazolium bis trifluoromethylsulfonyl imide & -0.46 \\
\hline 1,3-diethoxyimidazolium hexafluoro phosphate & -0.68 \\
\hline 1,3-dihydroxyimidazolium bis trifluoromethylsulfonyl imide & -3.93 \\
\hline 1,3-dihydroxy-2-methylimidazolium bis trifluoromethylsulfonyl imide & -3.59 \\
\hline 1,3-dimethoxyimidazolium bis trifluoromethylsulfonyl imide & -0.68 \\
\hline 1,3-dimethoxyimidazolium hexafluoro phosphate & -0.63 \\
\hline 1,3-dimethoxy-2-methylimidazolium bis trifluoromethylsulfonyl imide & -0.11 \\
\hline 1,3-dimethoxy-2-methylimidazolium hexafluoro phosphate & 0.61 \\
\hline 1,3-dimethylimidazolium dimethyl phosphate & -2.64 \\
\hline 1,3-dimethylimidazolium methansulfonate & -0.52 \\
\hline 1,3-dimethylimidazolium methyl sulfate & -0.92 \\
\hline 1,2-dimethyl-3-propylimidazolium bis trifluoromethylsulfonyl imide & 0.24 \\
\hline 1,2-dimethyl-3-propylimidazolium tris trifluoromethylsulfonyl methide & 0.67 \\
\hline 1-dodecyl-3-methylimidazolium iodine & -1.98 \\
\hline 1-ethyl-2,3-dimethylimidazolium tetrafluroborate & -1.44 \\
\hline 1-ethyl-2,3-dimethylimidazolium chlorine & -6.07 \\
\hline 1-ethyl-2,3-dimethylimidazolium ethyl sulfate & -1.84 \\
\hline 1-ethyl-2,3-dimethylimidazolium hexafluoro phosphate & 1.02 \\
\hline 1-ethyl-2,3-dimethylimidazolium trifluoromethanesulfonate & -1.14 \\
\hline 1-ethyl-3-methylimidazolium acetate & -4.84 \\
\hline 1-ethyl-3-methylimidazolium aminoacetate & -4.48 \\
\hline
\end{tabular}




\begin{tabular}{|c|c|}
\hline 1-ethyl-3-methylimidazolium aminopropionate & -4.32 \\
\hline 1-ethyl-3-methylimidazolium bis-pentafluoroethylsulfonyl imide & 0.02 \\
\hline 1-ethyl-3-methylimidazolium bis trifluoromethylsulfonyl imide & -0.16 \\
\hline 1-ethyl-3-methylimidazolium bromine & -3.84 \\
\hline 1-ethyl-3-methylimidazolium chlorine & -5.45 \\
\hline 1-ethyl-3-methylimidazolium dibutyl phosphate & -3.06 \\
\hline 1-ethyl-3-methylimidazolium dicyanamide & -1.30 \\
\hline 1-ethyl-3-methylimidazolium diethyl phosphate & -3.06 \\
\hline 1-ethyl-3-methylimidazolium dimethyl phosphate & -3.20 \\
\hline 1-ethyl-3-methylimidazolium hexafluoro phosphate & 0.39 \\
\hline 1-ethyl-3-methylimidazolium hydrogen sulfate & -13.60 \\
\hline 1-ethyl-3-methylimidazolium hydroxide & -9.81 \\
\hline 1-ethyl-3-methylimidazolium iodine & -2.23 \\
\hline 1-ethyl-3-methylimidazolium lactate & -4.27 \\
\hline 1-ethyl-3-methylimidazolium methansulfonate & -0.79 \\
\hline 1-ethyl-3-methylimidazolium methyl sulfate & -1.35 \\
\hline 1-ethyl-3-methylimidazolium ethyl sulfate & -1.37 \\
\hline 1-ethyl-3-methylimidazolium nitrate & -2.11 \\
\hline 1-ethyl-3-methylimidazolium tetrachloroaluminate & -6.08 \\
\hline 1-ethyl-3-methylimidazolium tetrafluroborate & -0.89 \\
\hline 1-ethyl-3-methylimidazolium 1,1,2,2-tetrafluoroethane sulfonate & -0.76 \\
\hline 1-ethyl-3-methylimidazolium thiocyanate & -1.16 \\
\hline 1-ethyl-3-methylimidazolium tosylate & -2.03 \\
\hline 1-ethyl-3-methylimidazolium trifluoromethanesulfonate & -0.80 \\
\hline 1-hexyl-3-methylimidazolium bis trifluoromethylsulfonyl imide & -0.17 \\
\hline 1-hexyl-3-methylimidazolium chlorine & -4.43 \\
\hline 1-hexyl-3-methylimidazolium hexafluoro phosphate & -0.19 \\
\hline 1-hexyl-3-methylimidazolium iodine & -1.82 \\
\hline 1-hexyl-3-methylimidazolium tetrafluroborate & -0.94 \\
\hline 1-hexyl-3-methylimidazolium trifluoromethanesulfonate & -0.82 \\
\hline
\end{tabular}




\begin{tabular}{|c|c|c|}
\hline & 1,2-hydroxyethyl-3-methylimidazolium dicyanamide & -1.18 \\
\hline & 1-methylimidazolium chlorine & -13.85 \\
\hline & 1-methylimidazolium hydrogen sulfate & -4.17 \\
\hline & 1-methyl-3-octylimidazolium chlorine & -4.80 \\
\hline & 1-methyl-3-octylimidazolium hexafluoro phosphate & 0.12 \\
\hline & 1-methyl-3-octylimidazolium tetrafluroborate & -1.16 \\
\hline & 1-methyl-3-octylimidazolium trifluoromethanesulfonate & -0.94 \\
\hline & 1-methyl-3-propylimidazolium iodine & -2.14 \\
\hline & 1-methyl-3-propylimidazolium methyl carbonate & -3.65 \\
\hline & 1-methyl-3-propylimidazolium methyl carbonate & -3.65 \\
\hline & 1-propyl-3-methylimidazolium bis trifluoromethylsulfonyl imide & -0.24 \\
\hline & 1,2,3-trimethylimidazolium methyl sulfate & -1.76 \\
\hline & Tetrabutylphosphonium methansulfonate & -1.03 \\
\hline & Tetrabutylphosphonium tetrafluroborate & -1.60 \\
\hline & Tetrabutylphosphonium p-toluenesulfonate & -2.71 \\
\hline & Tributylmethylphosphonium dibutyl phosphate & -3.91 \\
\hline & Tributylmethylphosphonium methyl sulfate & -1.98 \\
\hline$\Xi$ & Triethylmethylphosphonium dibutyl phosphate & -3.86 \\
\hline$\stackrel{\varrho}{1}$ & Trihexyltetradecylphosphonium bis trifluoromethylsulfonyl imide & 1.22 \\
\hline$=$ & Trihexyltetradecylphosphonium bis-2,4,4-trimethylpentyl phosphinate & -4.63 \\
\hline & Trihexyltetradecylphosphonium bromine & -4.01 \\
\hline & Trihexyltetradecylphosphonium chlorine & -5.68 \\
\hline & Trihexyltetradecylphosphonium dicyanamide & -1.69 \\
\hline & 3-triphenylphosphonio-propane-1-sulfonate & -1.08 \\
\hline & 3-triphenylphosphonio-propane-1-sulfonicacid tosylate & -1.67 \\
\hline & 1-butyl-3-methylpyridinium bis trifluoromethylsulfonyl imide & 0.11 \\
\hline & 1-butyl-3-methylpyridinium chlorine & -4.93 \\
\hline 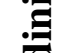 & 1-butyl-3-methylpyridinium hexafluoro phosphate & 0.53 \\
\hline . & 1-butyl-3-methylpyridinium iodine & -2.15 \\
\hline & 1-butyl-3-methylpyridinium tetrafluroborate & -1.17 \\
\hline
\end{tabular}




\begin{tabular}{|l|l|}
\hline 1-butylpyridinium bromine & -2.89 \\
\hline 1,3-cyanopropyl pyridinium chlorine & -3.92 \\
\hline 1-ethylpyridinium tetrafluroborate & -0.72 \\
\hline 3-methyl-1-propylpyridinium bis trifluoromethylsulfonyl imide & 0.07 \\
\hline 1-butyl-1-methylpyrrolidinium bis trifluoromethylsulfonyl imide & 0.28 \\
\hline 1-butyl-1-methylpyrrolidinium bromine & -4.24 \\
\hline 1-butyl-1-methylpyrrolidinium chlorine & -5.80 \\
\hline 1-butyl-1-methylpyrrolidinium dicyanamide & -2.02 \\
\hline 1-butyl-1-methylpyrrolidinium iodine & -2.68 \\
\hline 1-butyl-1-methylpyrrolidinium methyl carbonate & -4.49 \\
\hline 1-butyl-1-methylpyrrolidinium tetrafluroborate & -1.49 \\
\hline 1-butyl-1-methylpyrrolidinium trifluoromethanesulfonate & -1.16 \\
\hline 1-ethyl-1-methylpyrrolidinium bis trifluoromethylsulfonyl imide & 0.25 \\
\hline 1-ethyl-1-methylpyrrolidinium bromine & -4.67 \\
\hline 1-ethyl-1-methylpyrrolidinium hexafluoro phosphate & 1.20 \\
\hline 1-ethyl-1-methylpyrrolidinium tetrafluroborate & -1.50 \\
\hline 1-butyl-3-methylimidazolium acetate & -4.33 \\
\hline 1-butyl-3-methylimidazolium bis trifluoromethylsulfonyl imide & -0.21 \\
\hline 1-butyl-3-methylimidazolium chlorine & -4.60 \\
\hline 1-butyl-3-methylimidazolium hydrogen sulfate & -12.65 \\
\hline 1-butyl-3-methylimidazolium tetrafluroborate & -0.85 \\
\hline 1-butyl-3-methylimidazolium methansulfonate & -0.79 \\
\hline 1-butyl-3-methylimidazolium methyl sulfate & -1.25 \\
\hline 1-butyl-3-methylimidazolium thiocyanate & -1.05 \\
\hline 1-butyl-3-methylimidazolium trifluoromethanesulfonate & -0.80 \\
\hline 1-ethyl-2,3-dimethylimidazolium ethyl sulfate & -1.84 \\
\hline 1-ethyl-3-methylimidazolium bis trifluoromethylsulfonyl imide & -0.16 \\
\hline 1-ethyl-3-methylimidazolium acetate & -4.84 \\
\hline 1-ethyl-3-methylimidazolium chlorine & -5.45 \\
\hline
\end{tabular}




\begin{tabular}{|c|c|c|}
\hline & 1-ethyl-3-methylimidazolium diethyl phosphate & -3.06 \\
\hline & 1-ethyl-3-methylimidazolium hydrogen sulfate & -13.60 \\
\hline & 1-ethyl-3-methylimidazolium hydroxide & -9.81 \\
\hline & 1-ethyl-3-methylimidazolium methansulfonate & -0.79 \\
\hline & 1-ethyl-3-methylimidazolium tetrachloroaluminate & -6.08 \\
\hline & 1-ethyl-3-methylimidazolium tetrafluroborate & -0.89 \\
\hline & 1-ethyl-3-methylimidazolium thiocyanate & -1.16 \\
\hline & 1-ethyl-3-methylimidazolium trifluoromethanesulfonate & -0.80 \\
\hline & 1-methylimidazolium chlorine & -13.85 \\
\hline & 1-methylimidazolium hydrogen sulfate & -4.17 \\
\hline & 1-propyl-3-methylimidazolium bis trifluoromethylsulfonyl imide & -0.24 \\
\hline & 1,2,3-trimethylimidazolium methyl sulfate & -1.76 \\
\hline$\frac{\Xi}{8}$ & Choline acetate & -3.87 \\
\hline$\Xi$ & Cyclopropyldiphenylsulfonium tetrafluroborate & -1.43 \\
\hline 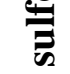 & Triethylsulfonium bis trifluoromethylsulfonyl imide & 0.07 \\
\hline & 1-butyl-1-methylpiperidinium bis trifluoromethylsulfonyl imide & 0.37 \\
\hline & 1-butyl-1-methylpiperidinium hexafluoro phosphate & 0.96 \\
\hline & 1-butyl-1-methylpiperidinium tetrafluroborate & -1.54 \\
\hline Ẽ & 4-ethyl-4-methylmorpholinium methyl carbonate & -3.81 \\
\hline & 1,2,3-tris-diethylamino cyclopropenylium bis trifluoromethylsulfonyl imide & -1.12 \\
\hline & 1,2,3-tris-diethylamino cyclopropenylium dicyanamide & -4.30 \\
\hline & Benzyldimethyltetradecylammonium chlorine & -5.12 \\
\hline & Butyltrimethylammonium bis trifluoromethylsulfonyl imide & 0.04 \\
\hline 严 & Diethylmethyl-2-methoxyethyl-ammonium bis trifluoromethylsulfonyl imide & 0.12 \\
\hline 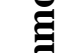 & Ethyldimethylpropylammonium bis trifluoromethylsulfonyl imide & 0.15 \\
\hline & 2-hydroxyethyl-trimethylammonium lactate & -3.43 \\
\hline & Methyltrioctadecylammonium bromine & -3.66 \\
\hline
\end{tabular}




\begin{tabular}{|l|c|}
\hline Methyl-trioctylammonium bis trifluoromethylsulfonyl imide & 0.99 \\
\hline Methyl-trioctylammonium hydrogen sulfate & -10.00 \\
\hline Methyl-trioctylammonium thiosalicylate & -4.21 \\
\hline Tetrabutylammonium benzoate & -5.56 \\
\hline Tetrabutylammonium bis trifluoromethylsulfonyl imide & 0.88 \\
\hline Tetrabutylammonium heptadecafluorooctanesulfonate & -0.37 \\
\hline Tetrabutylammonium hydroxide & -12.36 \\
\hline Tetrabutylammonium nitrite & -4.97 \\
\hline Tetrabutylammonium methansulfonate & -1.15 \\
\hline Tetrabutylammonium nonafluorobutanesulfonate & -0.64 \\
\hline Tetrabutylammonium succinimide & -4.78 \\
\hline Tetrabutylammonium thiophenolate & -3.56 \\
\hline Tetrabutylammonium triiodide & 2.10 \\
\hline Tetraethylammonium trifluoromethanesulfonate & -1.23 \\
\hline Tetraheptylammonium bromine & -4.14 \\
\hline Tetraheptylammonium chlorine & -5.77 \\
\hline Tetrahexylammonium hydrogen sulfate & -9.99 \\
\hline Tetrahexylammonium iodine & -2.68 \\
\hline Tetrahexylammonium tetrafluroborate & -1.43 \\
\hline Tetrakis-decyl-ammonium bromine & -4.09 \\
\hline Tetramethylammonium hydroxide & -13.40 \\
\hline Tetraoctylammonium chlorine & -5.89 \\
\hline Tetrapentylammonium bromine & -4.43 \\
\hline Tributylmethylammonium chlorine & -5.70 \\
\hline Tributylmethylammonium dibutyl phosphate & -3.79 \\
\hline Tributylmethylammonium methyl carbonate & -4.61 \\
\hline Tributylmethylammonium methyl sulfate & -1.91 \\
\hline Triethylmethylammonium dibutyl phosphate & -3.84 \\
\hline Triethylmethylammonium methyl carbonate & -4.70 \\
\hline Tris-2-hydroxyethy-methylammonium methyl sulfate & -0.10 \\
\hline
\end{tabular}


Table S2. The electron donor orbitals, electron acceptor orbitals, the corresponding second order interaction energies $E^{(2)}\left(\mathrm{KJ} \mathrm{mol}^{-1}\right)$ and corresponding bond lengths of IL and EDAB/IL complexes.

\begin{tabular}{|c|c|c|c|c|}
\hline Complex & Donor & Acceptor & $E^{(2)}\left(\mathrm{KJ} \mathrm{mol}^{-1}\right)$ & $\begin{array}{c}\text { Bond length between donor and } \\
\text { acceptor }(\AA)\end{array}$ \\
\hline \multirow{14}{*}{ 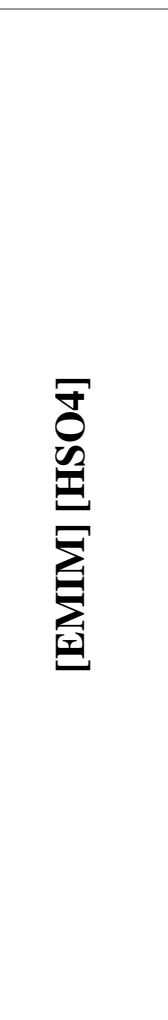 } & LP (1) O21 & $\mathrm{BD} *(1) \mathrm{C} 2$ - H9 & 9.12 & \multirow{3}{*}{2.00} \\
\hline & LP (2) O21 & $\mathrm{BD} *(1) \mathrm{C} 2$ - H9 & 24.05 & \\
\hline & LP (3) O21 & $\mathrm{BD}^{*}(1) \mathrm{C} 2-\mathrm{H} 9$ & 2.72 & \\
\hline & LP (1) O21 & $\mathrm{BD} *(1) \mathrm{C} 6-\mathrm{H} 12$ & 5.56 & \multirow{3}{*}{2.28} \\
\hline & LP (2) O21 & $\mathrm{BD} *(1) \mathrm{C} 6-\mathrm{H} 12$ & 1.17 & \\
\hline & LP (3) O21 & $\mathrm{BD} *(1) \mathrm{C} 6-\mathrm{H} 12$ & 1.25 & \\
\hline & LP (1) O22 & $\mathrm{BD}^{*}(1) \mathrm{C} 2$ - H9 & 3.30 & \multirow{3}{*}{2.22} \\
\hline & $\mathrm{LP}(2) \mathrm{O} 22$ & $\mathrm{BD} *(1) \mathrm{C} 2$ - H9 & 7.36 & \\
\hline & LP (3) O22 & $\mathrm{BD} *(1) \mathrm{C} 2-\mathrm{H} 9$ & 2.13 & \\
\hline & LP (1) O22 & $\mathrm{BD} *(1) \mathrm{C} 7-\mathrm{H} 16$ & 1.63 & \multirow{3}{*}{2.43} \\
\hline & $\mathrm{LP}(2) \mathrm{O} 22$ & $\mathrm{BD} *(1) \mathrm{C} 7-\mathrm{H} 16$ & 0.21 & \\
\hline & LP (3) O22 & $\mathrm{BD} *(1) \mathrm{C} 7-\mathrm{H} 16$ & 1.21 & \\
\hline & LP (1) O22 & $\mathrm{BD} *(1) \mathrm{C} 8-\mathrm{H} 17$ & 0.33 & \multirow{2}{*}{2.79} \\
\hline & LP (3) O22 & $\mathrm{BD} *(1) \mathrm{C} 8-\mathrm{H} 17$ & 0.59 & \\
\hline \multirow{12}{*}{ 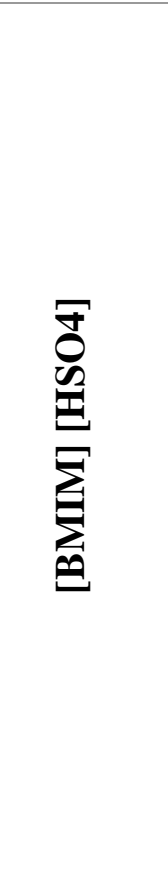 } & LP (1) O27 & $\mathrm{BD} *(1) \mathrm{C} 2-\mathrm{H} 9$ & 7.53 & \multirow{3}{*}{2.06} \\
\hline & LP (2) O27 & $\mathrm{BD}^{*}(1) \mathrm{C} 2$ - H9 & 16.18 & \\
\hline & LP (3) O27 & $\mathrm{BD} *(1) \mathrm{C} 2-\mathrm{H} 9$ & 5.52 & \\
\hline & LP (1) O27 & $\mathrm{BD} *(1) \mathrm{C} 6-\mathrm{H} 12$ & 4.47 & \multirow{2}{*}{2.37} \\
\hline & LP (2) O27 & BD*(1) C6 - H12 & 0.92 & \\
\hline & LP (1) O28 & $\mathrm{BD}^{*}(1) \mathrm{C} 2$ - H9 & 4.93 & \multirow{3}{*}{2.15} \\
\hline & LP (2) O28 & $\mathrm{BD}^{*}(1) \mathrm{C} 2$ - H9 & 12.38 & \\
\hline & LP (3) O28 & $\mathrm{BD}^{*}(1) \mathrm{C} 2-\mathrm{H} 9$ & 1.55 & \\
\hline & LP (1) O28 & BD*(1) C7 - H18 & 1.59 & \multirow{2}{*}{2.46} \\
\hline & LP (3) O28 & $\mathrm{BD} *(1) \mathrm{C} 7-\mathrm{H} 18$ & 0.88 & \\
\hline & LP (1) O28 & $\mathrm{BD}^{*}(1) \mathrm{C} 8-\mathrm{H} 19$ & 0.29 & \multirow{2}{*}{2.84} \\
\hline & LP (3) O28 & BD*(1) C8 - H19 & 0.42 & \\
\hline & LP (1) O21 & $\mathrm{BD} *(1) \mathrm{C} 2-\mathrm{H} 9$ & 0.29 & 2.64 \\
\hline
\end{tabular}




\begin{tabular}{|c|c|c|c|c|}
\hline \multirow{15}{*}{ 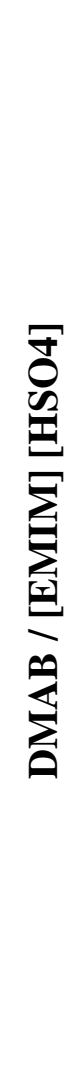 } & LP (2) O21 & $\mathrm{BD}^{*}(1) \mathrm{C} 2-\mathrm{H} 9$ & 0.21 & \\
\hline & LP (1) O21 & BD*(1) C6 - H12 & 0.33 & \multirow{2}{*}{2.72} \\
\hline & LP (2) O21 & $\mathrm{BD} *(1) \mathrm{C} 6-\mathrm{H} 12$ & 0.63 & \\
\hline & LP (1) O23 & $\mathrm{BD} *(1) \mathrm{C} 8-\mathrm{H} 18$ & 1.21 & \multirow{3}{*}{2.58} \\
\hline & LP (2) O23 & $\mathrm{BD} *(1) \mathrm{C} 8-\mathrm{H} 18$ & 1.17 & \\
\hline & LP (3) O23 & $\mathrm{BD} *(1) \mathrm{C} 8-\mathrm{H} 18$ & 2.97 & \\
\hline & LP (1) O22 & $\mathrm{BD}^{*}(1) \mathrm{C} 2-\mathrm{H} 9$ & 6.98 & \multirow{3}{*}{2.10} \\
\hline & LP (2) O22 & $\mathrm{BD} *(1) \mathrm{C} 2-\mathrm{H} 9$ & 7.78 & \\
\hline & LP (3) O22 & $\mathrm{BD} *(1) \mathrm{C} 2-\mathrm{H} 9$ & 7.07 & \\
\hline & LP (1) O22 & $\mathrm{BD} *(1) \mathrm{C} 7-\mathrm{H} 16$ & 1.25 & \multirow{3}{*}{2.54} \\
\hline & LP (2) O22 & $\mathrm{BD} *(1) \mathrm{C} 7-\mathrm{H} 16$ & 0.59 & \\
\hline & LP (3) O22 & BD*(1) C7 - H16 & 1.00 & \\
\hline & LP (1) O21 & $\mathrm{BD} *(1) \mathrm{N} 26-\mathrm{H} 30$ & 24.34 & \multirow{3}{*}{1.86} \\
\hline & LP (2) O21 & BD*(1) N26 - H30 & 31.66 & \\
\hline & LP (3) O21 & $\mathrm{BD}^{*}(1) \mathrm{N} 26-\mathrm{H} 30$ & 1.00 & \\
\hline \multirow{15}{*}{ 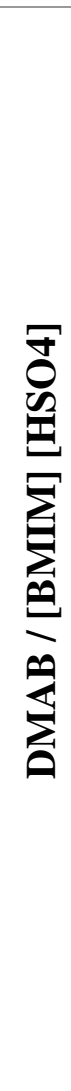 } & LP (1) O28 & $\mathrm{BD} *(1) \mathrm{C} 2-\mathrm{H} 9$ & 3.72 & \multirow{3}{*}{2.19} \\
\hline & LP (2) O28 & $\mathrm{BD}^{*}(1) \mathrm{C} 2-\mathrm{H} 9$ & 10.66 & \\
\hline & LP (3) O28 & $\mathrm{BD}^{*}(1) \mathrm{C} 2$ - H9 & 1.05 & \\
\hline & LP (1) O28 & $\mathrm{BD} *(1) \mathrm{C} 6-\mathrm{H} 12$ & 4.47 & \multirow{2}{*}{2.27} \\
\hline & LP (3) O28 & BD*(1) C6 - H12 & 6.77 & \\
\hline & LP (1) O29 & $\mathrm{BD} *(1) \mathrm{C} 2-\mathrm{H} 9$ & 0.67 & \multirow{2}{*}{2.49} \\
\hline & LP (2) O29 & $\mathrm{BD} *(1) \mathrm{C} 2-\mathrm{H} 9$ & 1.71 & \\
\hline & LP (1) O29 & BD*(1) C7 - H18 & 0.46 & \multirow{3}{*}{2.62} \\
\hline & LP (2) O29 & $\mathrm{BD} *(1) \mathrm{C} 7-\mathrm{H} 18$ & 0.50 & \\
\hline & LP (3) O29 & BD*(1) C7 - H18 & 0.29 & \\
\hline & LP (1) O29 & $\mathrm{BD} *(1) \mathrm{C} 8-\mathrm{H} 19$ & 0.84 & \multirow{2}{*}{2.57} \\
\hline & LP (3) O29 & $\mathrm{BD} *(1) \mathrm{C} 8-\mathrm{H} 19$ & 1.88 & \\
\hline & LP (1) O27 & BD*(1) N32 - H36 & 29.48 & \multirow{3}{*}{1.81} \\
\hline & LP (2) O27 & BD*(1) N32 - H36 & 8.49 & \\
\hline & LP (3) O27 & $\mathrm{BD}^{*}(1) \mathrm{N} 32-\mathrm{H} 36$ & 33.46 & \\
\hline
\end{tabular}

